\title{
Astrophysical Aspects of Neutrino Dynamics in Ultradegenerate Quark Gluon Plasma
}

\author{
Souvik Priyam Adhya \\ Experimental High Energy Physics and Applications Group, Variable Energy Cyclotron Center, 1/AF Bidhannagar, \\ Kolkata 700 064, India \\ Correspondence should be addressed to Souvik Priyam Adhya; souvikadhya2007@gmail.com
}

Received 20 July 2016; Revised 31 December 2016; Accepted 19 February 2017; Published 16 March 2017

Academic Editor: Sally Seidel

Copyright (C) 2017 Souvik Priyam Adhya. This is an open access article distributed under the Creative Commons Attribution License, which permits unrestricted use, distribution, and reproduction in any medium, provided the original work is properly cited. The publication of this article was funded by SCOAP $^{3}$.

\begin{abstract}
The cardinal focus of the present review is to explore the role of neutrinos originating from the ultradense core of neutron stars composed of quark gluon plasma in the astrophysical scenario. The collective excitations of the quarks involving the neutrinos through the different kinematical processes have been studied. The cooling of the neutron stars as well as pulsar kicks due to asymmetric neutrino emission has been discussed in detail. Results involving calculation of relevant physical quantities like neutrino mean free path and emissivity have been presented in the framework of non-Fermi liquid behavior as applicable to ultradegenerate plasma.
\end{abstract}

\section{Introduction}

Compact stars, in minutes or hours after their birth, rapidly cool down to temperatures $(T)$ of the order of $10^{9} \mathrm{~K}$ from upper bounds of $T \succsim 10^{11} \mathrm{~K}$. The neutrinos produced in direct reactions at the core are essentially transported to the surface leading to the cooling of the neutron star (NS) [17]. It is well known that the matter density in the core of the neutron stars could well exceed up to a few times the nuclear matter saturation density. Thus, in this context, we can expect comparable proportions of up, down, and strange quarks $[8,9]$ which are quite truly the ground state of QCD at finite baryon density. In addition, at such high densities, the constituents of matter are relativistic which compels one to describe such matter in the framework of non-Fermi liquid (NFL) behavior. However, the magnetic interaction is suppressed in powers of $(v / c)^{2}$ for nonrelativistic system. In fact, due to the presence of the magnetic contribution, the long range transverse interactions in deconfined degenerate quark matter give rise to the NFL behavior manifested in the appearance of anomalous contribution to the low temperature limit of the quark self-energy. The breakdown of the Fermi liquid (FL) picture has drawn substantial theoretical interest primarily due to the detection of the NFL behavior in normal state of superconductors [10-13] and in the systems of strongly correlated electrons [14-16]. In a contemporary system such as the quark gluon plasma, at the limit of low temperature, the chromomagnetostatic fields are unscreened unlike the high temperature case. Thus, we find a logarithmic singularity in the inverse group velocity. This singularity is removed by taking into account hard dense loop (HDL) approximations to the propagators $[17,18]$. This recently discovered phenomenon of NFL behavior relates itself to the modified quark dispersion relation. Therefore, for excitations close to the Fermi surface, NFL phenomena find important application in this realm of phase space. It has been shown that the NFL corrections to the quark selfenergy enhance the neutrino emissivity of ungapped quark matter which may exist at the core of neutron stars [19]. Like emissivity, in dense quark matter, the neutrino mean free path (MFP) also receives significant NFL corrections as has been demonstrated in [20]. In many calculations, such corrections appear in the modification of the phase space giving rise to appreciable contributions to the well known FL terms. Knowledge of the neutrino emissivity showing NFL behavior from quark cores from the neutrino mean free path (MFP) in compact stars is an essential prerequisite for a comprehensive understanding of a host of interesting phenomena such 
as the cooling of the star. In view of these contemporary investigations, we here plan to present the neutrino MFP and corresponding emissivity in normal degenerate quark matter beyond leading logarithmic order and compare it with the leading order (LO) results. Here, LO refers to the anomalous logarithmic term $T \log (1 / T)$ that occurs as the first term in the non-Fermi liquid contribution to the fermion self-energy. The quantities such as MFP and emissivity of neutrinos calculated with this term are called the LO corrections. Next to leading order (NLO) terms include all other terms beyond the LO that contain the fractional powers of $T$ and up to $\left(T^{3}\right) \log (1 / T)$. Quantities calculated with this correction are labeled as NLO corrections [21]. Knowing the specific heat of dense quark matter up to the order concerned, we investigate the cooling behavior of the neutron star with dense quark core. The important theoretical input required for the study of the emissivity and cooling is the neutrino MFP from such quark cores. In all these cases, the correction at the LO has been seen to involve $T \ln (1 / T)$ term which has been dubbed as anomalous corrections in many of the literatures [21-23]. In $[17,18]$, it has been shown that the fermion damping rate and energy loss receive significant NFL correction from the LO terms. Similar behavior has also been reported while investigating quantities like drag, diffusion coefficients, and thermal relaxation time of electrons for relativistic degenerate plasma [24-26]. In fact, the specific heat of the quark matter is also modified due to such corrections beyond the FL order [27]. In this review, we will also present the specific heat of degenerate quark matter which receives significant correction due to the presence of the high external magnetic field while including the NFL corrections. Such high magnetic fields are present at the core of the NS. Thus, the inclusion of the magnetic field in the specific heat in the case of interacting plasma and its effect on the kick velocity of the NS are studied in detail. The mechanism for the generation of such phenomenal kicks is related to the polarization of the electrons leading to neutrino emission in a preferred direction. The magnetic field strength required has to be equal to or greater than $B_{\text {cric }}=m_{i}^{2} c^{3} /\left(q_{i} \hbar\right)$, where $m_{i}$ and $q_{i}$ are the mass and charge of the electron. This leads to the formation of the neutrino and antineutrino emission cones which give rise to polarized neutrino emission opposite to the direction of the magnetic field producing the recoil velocity. We have used the electron polarization for different conditions of magnetic field and kick velocities which has been studied recently by Sagert et al. [28, 29], where the dependence of the kick velocity on the quark phase temperature and radius of the star with varying quark chemical potentials has also been investigated. In fact, experimental efforts like ROSAT, CHANDRA, and XMM have done a lot of measurements to understand the properties of the neutron stars $[30,31]$. Thus, at initial times, the dominant cooling mechanism is by neutrinos only and, at later times, by thermal emission. In addition, if color superconductivity effects are taken into account, the neutrino emissivity and specific heat are then suppressed exponentially by a factor $\exp (-\Delta / T)$, where $\Delta$ is the quasi-particle gap in the color-flavor locked (CFL) phase. However, the presence of the color superconductivity might lower the pulsar acceleration $[28,32]$.
This review is based upon the recent works $[19,20,28$, 29, 33-35] where the authors have explored various aspects of the neutrino dynamics in reactions involving ultradegenerate quark matter. In this review, we discuss the mean free path of the neutrinos and emissivity of neutrinos from neutron star in Sections 2 and 3, respectively. In addition, we will present results to see how the cooling is affected when one considers the anomalous corrections into the calculable physical quantities in Section 3. In Section 4, we will extend this formalism and introduce the effect of the external magnetic field to calculate the specific heat capacity of degenerate quark matter. This quantity directly influences the kick velocity of pulsars (i.e., rapidly rotating neutron stars) which arises due to asymmetric neutrino emission. Finally, we will present the results and conclusions in Section 5.

\section{Neutrino Mean Free Path}

2.1. The Case of Degenerate Neutrinos. The dominant contribution to the mean free path as well as emission of neutrinos is given by the quark analog of $\beta$ decay and the electron capture reaction producing neutrinos [36-39]. The degenerate neutrinos refer to the case where the neutrino chemical potential $\left(\mu_{v}\right)$ is much larger than the temperature:

$$
\begin{aligned}
& d+v_{e} \longrightarrow u+e^{-}, \\
& u+e^{-} \longrightarrow d+v_{e} .
\end{aligned}
$$

In addition, the quark-neutrino scattering process is given by

$$
q_{i}+v_{e}\left(\bar{\nu}_{e}\right) \longrightarrow q_{i}+v_{e}\left(\bar{\nu}_{e}\right)
$$

The corresponding mean free paths are denoted by $l_{\text {mean }}^{\text {abs }}$ and $l_{\text {mean }}^{\text {scatt }}$ which are subsequently combined to obtain the total mean free path of the neutrinos:

$$
\frac{1}{l_{\text {mean }}^{\text {total }}}=\frac{1}{l_{\text {mean }}^{\mathrm{abs}}}+\frac{1}{l_{\text {mean }}^{\text {scatt }}} .
$$

The MFP of the neutrinos is related to the total interaction rate due to neutrino emission which is averaged over the initial quark spins and eventually summed over the final state phase space and spins. It is given by [36]

$$
\begin{aligned}
& \frac{1}{l_{\text {mean }}^{\text {abs }}\left(E_{v}, T\right)}=\frac{g^{\prime}}{2 E_{v}} \int \frac{d^{3} p_{d}}{(2 \pi)^{3}} \frac{1}{2 E_{d}} \int \frac{d^{3} p_{u}}{(2 \pi)^{3}} \frac{1}{2 E_{u}} \int \frac{d^{3} p_{e}}{(2 \pi)^{3}} \\
& \cdot \frac{1}{2 E_{e}}(2 \pi)^{4} \delta^{4}\left(P_{d}+P_{v}-P_{u}-P_{e}\right) \times|M|^{2} \\
& \cdot\left\{n\left(p_{d}\right)\left[1-n\left(p_{u}\right)\right]\left[1-n\left(p_{e}\right)\right]\right. \\
& \left.+n\left(p_{u}\right) n\left(p_{e}\right)\left[1-n\left(p_{d}\right)\right]\right\},
\end{aligned}
$$

where $g^{\prime}$ is the spin and color degeneracy, taken as 6, and $E$, $p$, and $n_{p}$ are the energy, momentum, and distribution function for the corresponding particle. The squared invariant amplitude $|M|^{2}$ is given by $|M|^{2}=64 G^{2} \cos ^{2} \theta_{c}\left(P_{d} \cdot P_{v}\right)\left(P_{u}\right.$. $\left.P_{e}\right)$. Here, $G \simeq 1.435 \times 10^{-49} \mathrm{erg}-\mathrm{cm}^{3}$ is the weak coupling 
constant. The interaction involving strange quark is Cabibbo suppressed due to which the neutrino emission from strange quark matter can be neglected. We here consider the case of degenerate neutrinos (i.e., when $\mu_{\gamma} \gg T$ ). So, in this case, both direct (1) and inverse (2) processes are relevant. Therefore, the $\beta$ equilibrium condition becomes $\mu_{d}+\mu_{v}=$ $\mu_{u}+\mu_{e}$. Now, the momentum integration, $d^{3} p_{d}$ and $d^{3} p_{u}$, can be calculated as

$$
\begin{aligned}
& d^{3} p_{d}=2 \pi \frac{p_{f}(d)}{p_{f}(\nu)} p d p \frac{d p_{d}}{d \omega} d \omega, \\
& d^{3} p_{u}=2 \pi \frac{p_{f}(u) p_{f}(e)}{p} d E_{e} \frac{d p_{u}}{d \omega} d \omega,
\end{aligned}
$$

where we define $p \equiv\left|p_{d}+p_{\nu}\right|=\left|p_{u}+p_{e}\right| \cdot d p(\omega) / d \omega$ can be evaluated from the modified dispersion relation as follows [40]:

$$
\frac{d p(\omega)}{d \omega}=\left(1-\frac{\partial \operatorname{Re} \Sigma_{+}(\omega)}{\partial \omega}\right) \frac{E_{p(\omega)}}{p(\omega)} .
$$

We use the explicit form of the real part of the quark selfenergy in the ultradegenerate limit as found in $([21,27,33])$ which reads as follows:

$$
\begin{aligned}
& \Sigma(\omega)=-g^{2} C_{F} m\left\{\frac{\varepsilon}{12 \pi^{2} m}\left[\log \left(\frac{4 \sqrt{2} m}{\pi \varepsilon}\right)+1\right]\right. \\
& +\frac{i \varepsilon}{24 \pi m}+\frac{2^{1 / 3} \sqrt{3}}{45 \pi^{7 / 3}}\left(\frac{\varepsilon}{m}\right)^{5 / 3}(\operatorname{sgn}(\varepsilon)-\sqrt{3} i) \\
& +\frac{i}{64 \sqrt{2}}\left(\frac{\varepsilon}{m}\right)^{2}-20 \frac{2^{2 / 3} \sqrt{3}}{189 \pi^{11 / 3}}\left(\frac{\varepsilon}{m}\right)^{7 / 3} \\
& \cdot(\operatorname{sgn}(\varepsilon)+\sqrt{3} i) \\
& -\frac{6144-256 \pi^{2}+36 \pi^{4}-9 \pi^{6}}{864 \pi^{6}}\left(\frac{\varepsilon}{m}\right)^{3} \\
& \left.\cdot\left[\log \left(\frac{0.928 m}{\varepsilon}\right)-\frac{i \pi \operatorname{sgn}(\varepsilon)}{2}\right]+\mathcal{O}\left(\left(\frac{\varepsilon}{m}\right)^{11 / 3}\right)\right\}
\end{aligned}
$$

where $\varepsilon=(\omega-\mu) \sim T$, where NFL effects dominate. Neglecting the quark-quark interactions, the leading order result is obtained as $[20,33]$

$$
\begin{aligned}
& \left.\frac{1}{l_{\text {mean }}^{\mathrm{abs}, D}}\right|_{\mathrm{LO}} \\
& \simeq \frac{2}{3 \pi^{5}} G_{F}^{2} C_{F} \cos ^{2} \theta_{c} \frac{\mu_{e}^{3}}{\mu_{v}^{2}}\left[1+\frac{1}{2}\left(\frac{\mu_{e}}{\mu}\right)+\frac{1}{10}\left(\frac{\mu_{e}}{\mu}\right)^{2}\right] \\
& \times\left[\left(E_{\nu}-\mu_{\nu}\right)^{2}+\pi^{2} T^{2}\right](g \mu)^{2} \log \left(\frac{4 g \mu}{\pi^{2} T}\right) .
\end{aligned}
$$

The NLO result is evaluated as $[33,35,41]$

$$
\begin{aligned}
& \left.\frac{1}{l_{\text {mean }}^{\mathrm{abs} D}}\right|_{\mathrm{NLO}} \simeq \frac{8}{\pi^{3}} G_{F}^{2} C_{F} \cos ^{2} \theta_{c} \frac{\mu_{e}^{3}}{\mu_{\nu}^{2}}\left[1+\frac{1}{2}\left(\frac{\mu_{e}}{\mu}\right)\right. \\
& \left.+\frac{1}{10}\left(\frac{\mu_{e}}{\mu}\right)^{2}\right]\left[\left(E_{v}-\mu_{\nu}\right)^{2}+\pi^{2} T^{2}\right] \\
& \quad \times\left[a_{1} T^{2 / 3}(g \mu)^{4 / 3}+a_{2} T^{4 / 3}(g \mu)^{2 / 3}\right. \\
& \left.+a_{3}\left\{1-3 \log \left(\frac{0.209 g \mu}{T}\right)\right\} T^{2}\right],
\end{aligned}
$$

where the constants $a_{1}=0.015, a_{2}=-0.075$, and $a_{3}=$ -0.036 . For the evaluation of the constants, please refer to [33]. To arrive at the Fermi liquid result, one can use the free dispersion relation to arrive at [36]

$$
\begin{aligned}
\frac{1}{\left.l_{\text {mean }}^{\mathrm{abs}, D}\right|_{\mathrm{FL}}=} & \frac{4}{\pi^{3}} G_{F}^{2} \cos ^{2} \theta_{c} \\
& \cdot \frac{\mu^{2} \mu_{e}^{3}}{\mu_{v}^{2}}\left[1+\frac{1}{2}\left(\frac{\mu_{e}}{\mu}\right)+\frac{1}{10}\left(\frac{\mu_{e}}{\mu}\right)^{2}\right] \\
& \cdot\left[\left(E_{v}-\mu_{v}\right)^{2}+\pi^{2} T^{2}\right] .
\end{aligned}
$$

Quarks and electrons are considered to be massless in this framework, and the chemical equilibrium condition gives $p_{f}(u)+p_{f}(e)=p_{f}(d)+p_{f}(\nu)$, which we use to derive (11). In addition, we have assumed that $\mu_{d} \sim \mu_{u}=\mu$. The MFP for the quark-neutrino scattering process, for each quark component of flavor $i(=u$ or $d)$, is calculated which will contribute to the total neutrino flux. Proceeding in a similar way, we obtain $[20,33,36-39,41]$

$$
\begin{aligned}
& \left.\frac{1}{l_{\text {mean }}^{\text {scatt } D}}\right|_{\mathrm{FL}}=\frac{3}{4 \pi} n_{q_{i}} G_{F}^{2} \times\left[\left(E_{v}-\mu_{\nu}\right)^{2}+\pi^{2} T^{2}\right] \Lambda\left(x_{i}\right), \\
& \left.\frac{1}{l_{\text {mean }}^{\text {scatt } D}}\right|_{\mathrm{LO}} \simeq \frac{1}{8 \pi^{3}} n_{q_{i}} C_{F} G_{F}^{2}\left[\left(E_{v}-\mu_{\nu}\right)^{2}+\pi^{2} T^{2}\right] \Lambda\left(x_{i}\right) \\
& \cdot g^{2} \log \left(\frac{4 g \mu}{\pi^{2} T}\right), \\
& \left.\frac{1}{l_{\text {mean }}^{\text {scatt }, D}}\right|_{\mathrm{NLO}} \simeq \frac{3}{2 \pi} n_{q_{i}} C_{F} G_{F}^{2}\left[\left(E_{v}-\mu_{v}\right)^{2}+\pi^{2} T^{2}\right] \Lambda\left(x_{i}\right) \\
& \cdot\left[a_{1} g^{4 / 3}\left(\frac{T}{\mu}\right)^{2 / 3}+a_{2} g^{2 / 3}\left(\frac{T}{\mu}\right)^{4 / 3}\right. \\
& \left.\quad+a_{3}\left\{1-3 \log \left(\frac{0.209 g \mu}{T}\right)\right\}\left(\frac{T}{\mu}\right)^{2}\right],
\end{aligned}
$$

where $n_{q_{i}}$ is the number density of quark of flavor $i$ and $\sigma_{0} \equiv 4 G_{F}^{2} m_{e}^{2} / \pi[38,39]$ and $\Lambda\left(x_{i}\right)$ is defined in [20] where $x_{i}=\mu_{v} / \mu_{q_{i}}$ if $\mu_{\nu}<\mu_{q_{i}}$ and $x_{i}=\mu_{q_{i}} / \mu_{\nu}$ if $\mu_{\nu}>\mu_{q_{i}}$. $C_{v_{i}}$ and $C_{A_{i}}$ are the vector and axial vector coupling constants which are given in detail in Table (II) of [36-39]. The contributions from the Fermi liquid (FL), LO, and NLO are added to obtain the MFP of the neutrinos. 
2.2. The Case of Nondegenerate Neutrinos. We now derive MFP for nondegenerate neutrinos (i.e., when $\mu_{v} \ll T$ ) beyond the Fermi liquid contribution. For nondegenerate neutrinos, the inverse process (2) is not considered as, for untrapped case, the reverse reaction is assumed to be zero. Hence, we neglect the second term in the curly braces of (5) [36]. Including the strong interactions between the quarks results in a nonvanishing squared matrix amplitude. The neutrino momentum in energy conserving relation due to the thermal production of the neutrinos can safely be neglected [36]. The Fermi liquid case, LO, and NLO are given as [20, 33, $36,37,41]$

$$
\begin{aligned}
& \left.\frac{1}{l_{\text {mean }}^{\mathrm{abs}, N D}}\right|_{\mathrm{FL}}=\frac{3 C_{F} \alpha_{s}}{\pi^{4}} G_{F}^{2} \cos ^{2} \theta_{c} \mu_{d} \mu_{u} \mu_{e} \frac{\left(E_{v}^{2}+\pi^{2} T^{2}\right)}{\left(1+e^{-\beta E_{v}}\right)}, \\
& \left.\frac{1}{l_{\text {mean }}^{\text {abs, ND }}}\right|_{\text {LO }} \simeq \frac{C_{F}^{2} \alpha_{s}}{2 \pi^{6}} G_{F}^{2} \cos ^{2} \theta_{c} \mu_{e} \frac{\left(E_{v}^{2}+\pi^{2} T^{2}\right)}{\left(1+e^{-\beta E_{\nu}}\right)}(g \mu)^{2} \\
& \cdot \log \left(\frac{4 g \mu}{\pi^{2} T}\right) \\
& \left.\frac{1}{l_{\text {mean }}^{\text {abs,ND }}}\right|_{\mathrm{NLO}} \simeq \frac{3 C_{F}^{2} \alpha_{s}}{\pi^{4}} G_{F}^{2} \cos ^{2} \theta_{c} \mu^{2} \mu_{e} \\
& \cdot \frac{\left(E_{v}^{2}+\pi^{2} T^{2}\right)}{\left(1+e^{-\beta E_{v}}\right)}\left[b_{1} g^{4 / 3}\left(\frac{T}{\mu}\right)^{2 / 3}+b_{2} g^{2 / 3}\left(\frac{T}{\mu}\right)^{4 / 3}\right. \\
& \left.+b_{3}\left\{1-3 \log \left(\frac{0.209 g \mu}{T}\right)\right\}\left(\frac{T}{\mu}\right)^{2}\right] \text {, }
\end{aligned}
$$

where the constants are evaluated as $b_{1}=0.03, b_{2}=-0.149$, and $b_{3}=-0.073$ [33]. In the nondegenerate case, the FL term is proportional to $\alpha_{s}$ and is not the same as the free Fermi gas. However, for the degenerate case, the FL term contributes at the order $\left(\alpha_{s}\right)^{0}$. The difference arises due to the evaluation of the squared matrix element for the nondegenerate case where $v \cdot p_{e} \sim C_{F} \alpha_{s} \mu_{e} / \pi[19]$.

Similarly, for the scattering of nondegenerate neutrinos in quark matter with appropriate phase space corrections, we obtain $[20,33,36-39,41]$

$$
\begin{aligned}
& \left.\frac{1}{l_{\text {mean }}^{\text {scatt } N D}}\right|_{\mathrm{FL}}=\frac{C_{V_{i}}^{2}+C_{A_{i}}^{2}}{5 \pi} n_{q_{i}} G_{F}^{2} \frac{E_{v}^{3}}{\mu}, \\
& \left.\frac{1}{l_{\text {mean }}^{\text {scatt } N D}}\right|_{\mathrm{LO}} \simeq \frac{C_{V_{i}}^{2}+C_{A_{i}}^{2}}{30 \pi^{3}} n_{q_{i}} G_{F}^{2} C_{F} \frac{E_{v}^{3}}{\mu} g^{2} \log \left(\frac{4 g \mu}{\pi^{2} T}\right), \\
& \left.\frac{1}{l_{\text {mean }}^{\text {scatt } N D}}\right|_{\mathrm{NLO}} \simeq\left(C_{V_{i}}^{2}+C_{A_{i}}^{2}\right) n_{q_{i}} G_{F}^{2} C_{F}\left[b_{1}^{\prime} \frac{T^{2 / 3} g^{4 / 3}}{\mu^{5 / 3}}\right. \\
& \left.+b_{2}^{\prime} \frac{T^{4 / 3} g^{2 / 3}}{\mu^{7 / 3}}+b_{3}^{\prime}\left\{1-3 \log \left(\frac{0.209 g \mu}{T}\right)\right\}\left(\frac{T^{2}}{\mu^{3}}\right)\right],
\end{aligned}
$$

where $b_{1}^{\prime}=0.002, b_{2}^{\prime}=-0.009$, and $b_{3}^{\prime}=-0.005$ [33]. Here, it has been assumed that $m_{q_{i}} / p_{f_{i}} \ll 1$. Thus, the total MFP for nondegenerate neutrinos is obtained by summing up the contributions from the absorption and scattering parts to get the expression of the MFP of the nondegenerate neutrinos up to the NLO terms.

\section{Neutrino Emissivity}

In this section, we focus on the calculation of the neutrino emissivity from the neutrino MFP. For our calculation, we consider the MFP of nondegenerate neutrinos. The total emissivity of the nondegenerate neutrinos is obtained by multiplying the neutrino energy with the inverse of the MFP with appropriate factors and integrated over the neutrino momentum given by

$$
\varepsilon=\int \frac{d^{3} p_{v}}{(2 \pi)^{3}} E_{v} \frac{1}{l\left(-E_{v}, T\right)} .
$$

The total NFL correction can be obtained as

$$
\varepsilon-\varepsilon_{0}=\varepsilon_{\mathrm{LO}}+\varepsilon_{\mathrm{NLO}},
$$

where

$$
\varepsilon_{0} \simeq \frac{457}{630} G_{F}^{2} \cos ^{2} \theta_{c} \alpha_{s} \mu_{e} T^{6} \mu^{2}
$$

is the usual Fermi liquid contribution which agrees with the result presented in [36]. At the LO, we have obtained [19, 33, 41]

$$
\varepsilon_{\mathrm{LO}} \simeq \frac{457}{3780} G_{F}^{2} \cos ^{2} \theta_{c} C_{F} \alpha_{s} \mu_{e} T^{6} \frac{(g \mu)^{2}}{\pi^{2}} \ln \left(\frac{4 g \mu}{\pi^{2} T}\right) .
$$

The NLO contribution to the neutrino emissivity is $[33,35,41]$

$$
\begin{aligned}
\varepsilon_{\mathrm{NLO}} & \simeq \frac{457}{315} G_{F}^{2} \cos ^{2} \theta_{c} C_{F} \alpha_{s} \mu_{e} T^{6}\left[c_{1} T^{2}\right. \\
+ & c_{2} T^{2 / 3}(g \mu)^{4 / 3}-c_{3} T^{4 / 3}(g \mu)^{2 / 3} \\
& \left.-c_{4} T^{2} \ln \left(\frac{0.656 g \mu}{\pi T}\right)\right]
\end{aligned}
$$

where the constants are $c_{1}=-0.035, c_{2}=0.015, c_{3}=0.075$, and $c_{4}=-0.109$ [33]. The NFL correction which appears in the phase space integral of the MFP [20] and gradually in the expression of the emissivity [19] is actually related to the unscreened magnetic/transverse interaction. The factor of $T^{6}$ can be understood as one power of $T$ is obtained from phase space integral of a degenerate fermion and $T^{3}$ from the phase space integral of the neutrino. One power of $T$ from the energy conserving $\delta$ function is canceled by a power from the emitted neutrino energy. For excitations lying close to the Fermi surface, the angular integrals provide no temperature dependence.

3.1. Emissivity of Neutrinos from Analogous Processes. In addition to the abovementioned quark direct process, there are contributions from other processes in different domain of density and momentum of the constituent particles. For example, the neutrino emissivity from modified quark URCA process is given as [36]

$$
\varepsilon^{q \text { URCA }} \sim \alpha_{c}^{2} G_{F}^{2} \cos ^{2} \theta_{C} p_{F}(q)\left(k_{B} T\right)^{8} .
$$


In lower densities, dominant neutrino emission mechanisms are the one-nucleon processes (e.g., $n \rightarrow p e \bar{v}$ ), called nucleon direct URCA (DU) reactions. Their emissivity is [36]

$$
\varepsilon^{\mathrm{DU}} \sim 10^{27} \times T_{9}^{6}\left(\frac{n}{n_{0}}\right)^{2 / 3} \theta\left(n-n_{c}^{\mathrm{DU}}\right) \mathrm{erg} / \mathrm{cm}^{3} \mathrm{~s},
$$

where $n$ is the nucleon density measured in units of the nuclear matter saturation density $n_{0}$. The DU processes are operative only when the proton fraction exceeds a critical value of $11-14 \%$. Another similar process of quite interest is the two-nucleon interactions (in the presence of a bystander particle) (e.g., $n n \rightarrow n p e \bar{\nu}$ ), called modified Urca (MU) processes with the emissivity [36]

$$
\varepsilon^{\mathrm{MU}} \sim 10^{21} \times T_{9}^{8}\left(\frac{n}{n_{0}}\right)^{2 / 3} \mathrm{erg} / \mathrm{cm}^{3} \mathrm{~s} .
$$

However, other different processes, the nuclear Bremsstrahlung reactions, such as $n n \rightarrow n n \nu \bar{\nu}(\mathrm{nB}), n p \rightarrow$ $n p \nu \bar{v}(\mathrm{npB})$, and $p p \rightarrow p p \nu \bar{\nu}(\mathrm{pB})$, have an order of magnitude smaller emission rates than the MU processes. In addition, quark matter at asymptotically high density is in the color-flavor locked (CFL) phase [42] of color superconductivity (CSC). The CSC phases at moderate density are still unclear due to the complicated nonperturbative effect. Various candidates have been proposed, such as two-flavor CSC (2SC) [43], gapless 2SC [44, 45], gapless CFL (gCFL) [46], crystalline CSC [47], and spin-1 CSC [48-54]. Neutrino emission from CSC quark matter is exponentially suppressed at low temperature, if the quasi-particle spectra are fully gapped [55-60]. Similar to nuclear matter, the pair breaking and recombination effect results in an emissivity of $\epsilon \sim T^{7}$ at temperature close to $T_{c}$ [61]. The emissivity behaves as $\epsilon \sim T^{5.5}$ for gCFL [55] and $\epsilon \sim T^{6}$ for g2SC (see, e.g., Table 1 of [19]).

\section{Cooling Behavior of Neutron Star with Dense Quark Core}

The results of the MFP of neutrinos with the temperature have been presented in Figure 1. A quark chemical potential of $500 \mathrm{MeV}$ is considered for the process. This agrees well with the high density $\sim 6 \rho_{0}$ ( $\rho_{0}$ is the nuclear matter saturation density) at the core. We have taken $\mu_{e}=15 \mathrm{MeV}$ and $\alpha_{s}=0.1$. In Figure 1(a), we note that there is a considerable decrease in MFP of degenerate neutrinos due to NLO corrections over the Fermi liquid result. In Figure 1(b), LO correction is compared with the NLO correction and it is seen that the MFP with NLO correction is reduced marginally as compared to LO correction. Similar features have been observed in the case of the MFP of nondegenerate neutrinos as displayed in Figure 2. This modest difference in the MFP between NFL LO and NLO corrections also leads to marginal difference in the emissivity for the two cases. This is shown in Figure 3. These small reductions are reflected in the marginally enhanced emissivity of the nondegenerate neutrinos which has been shown in Figure 3(a). We note that there is a modest increase in the emissivity of the neutrinos. The complicated cooling equation has to be solved numerically as shown in Figure 3(b). The temperature of the neutron star with a quark matter core shows dependency on time. To analyze the cooling of the star $[31,62,63]$, the specific heat capacity of the quark matter core needs to be taken into consideration along with the emissivity via the cooling process $[19,36]$. We have considered the NFL contribution to the specific heat capacity to compute our results. We observe that the cooling of neutron star is marginally faster in case of NFL (NLO) as compared to the Fermi liquid result (shown in Figure 3). In addition, it is found that the cooling process from the quark core is much faster than the case where the nuclear matter is considered to be present at the core. This influence of magnetic interaction has a significant effect on the cooling behavior of the neutron star, which in the present case is modified due to incorporation of the NFL contribution beyond LO. This leads to marginally faster cooling of the neutron star due to neutrino emission URCA processes and the quark-neutrino scattering reaction.

\section{Pulsar Kick Velocity through Asymmetric Neutrino Emission}

The amount of pulsar acceleration depends on the polarization of the electron spin and the momenta. The kick velocity can be written as $[28,29]$

$$
d v=\frac{\chi}{M_{\mathrm{NS}}} \frac{4}{3} \pi R^{3} \varepsilon d t
$$

where the polarization fraction of the electrons has been denoted by $\chi$ and the neutrino emissivity by $\varepsilon$. Using the cooling equation,

$$
C_{v}^{B} d T=-\varepsilon d t
$$

One can rewrite $(23)$ in terms of the specific heat $\left(C_{v}\right)$ of the quark matter core. The influence of the exterior magnetic field on the specific heat of the quark matter in addition to the NFL effect can be explored in an interesting way. In the presence of a constant external magnetic field $(B)$ along the $z$-axis, the thermodynamic potential can be written as $[64,65]$

$$
\Omega^{B}=-\frac{g_{d} T|q| B}{2 \pi^{2}} \sum_{\nu=0}^{\infty} \int_{0}^{\infty} d p_{z} \log \left(1+e^{\beta(\mu-\epsilon)}\right),
$$

where $\epsilon=\sqrt{p_{z}^{2}+m^{2}+2 \nu|q| B}$ is the single particle energy eigenvalue, $g_{d}$ is the quark degeneracy, and $v=0,1,2, \ldots$. Thus, the specific heat computed from the thermodynamic potential is $[34,35]$

$$
\left.C_{v}\right|_{\mathrm{FL}} ^{B}=\frac{N_{C} N_{f} T m_{q}^{2}}{6}\left(\frac{B}{B_{\mathrm{cr}}^{q}}\right) .
$$

Including the effect of the NFL behavior in the specific heat capacity, through the in-medium quark dispersion relation,

$$
\omega=\left(E_{p(\omega)}+\operatorname{Re} \Sigma(\omega, p(\omega))\right),
$$




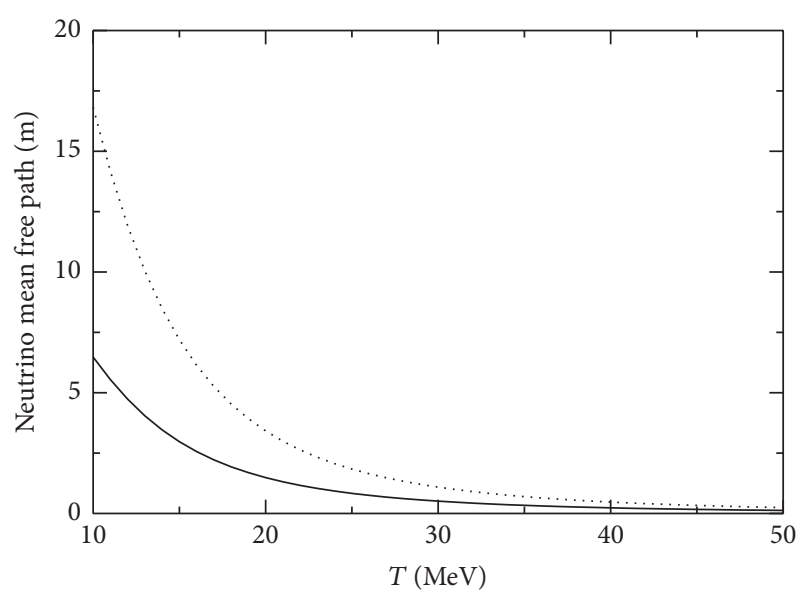

Fermi liquid result NFL (NLO)

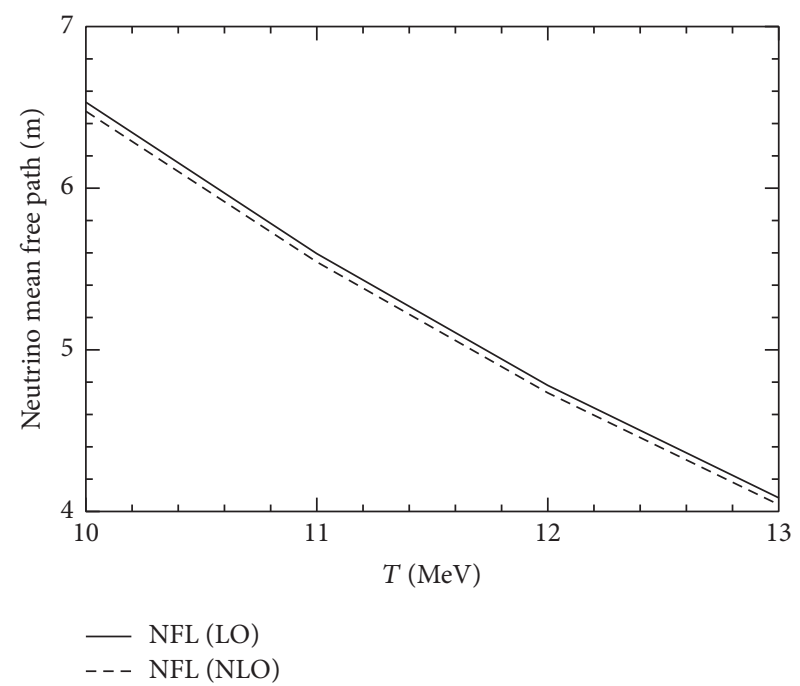

(b)

FIGURE 1: Mean free path of degenerate neutrinos. A comparison between the FL result and NLO corrections for the NFL effects has been shown in (a). The reduction of the MFP due to NLO corrections has been shown in (b).

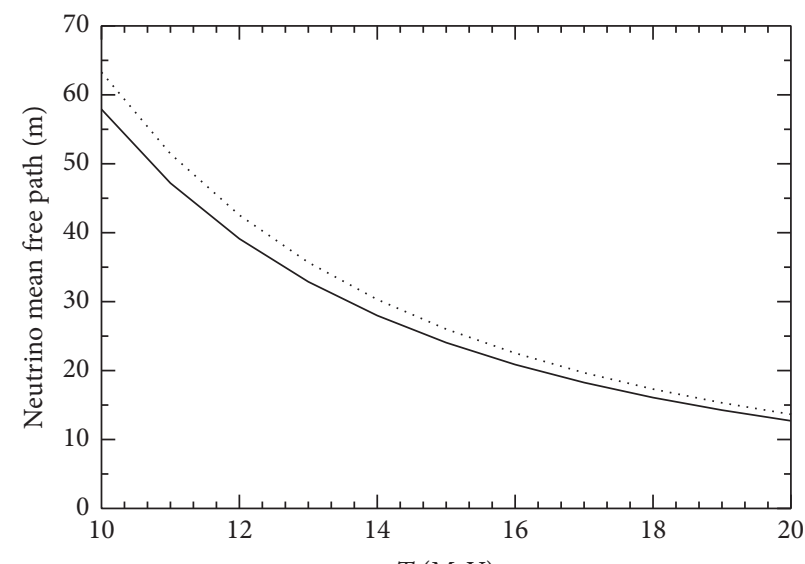

Fermi liquid result NFL (NLO)

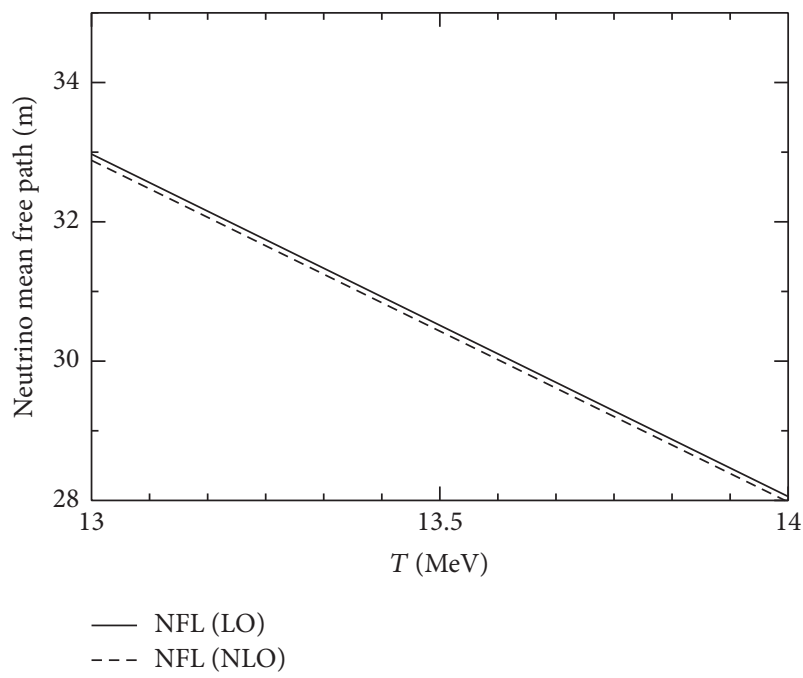

(b)

FIGURE 2: Mean free path of nondegenerate neutrinos. A comparison between the FL result and NLO corrections for the NFL effects has been presented in (a). The reduction of the MFP due to NLO corrections has been shown in (b).

where $\Sigma$ represents the one-loop quark quasi-particle selfenergy, and the LO correction is given as [34]

$$
\begin{aligned}
\left.C_{v}\right|_{\mathrm{LO}} ^{B} \simeq & \left(\frac{N_{C} N_{f} C_{f} \alpha_{s}}{36 \pi}\right) m_{q}^{2}\left(\frac{B}{B_{\mathrm{cr}}^{q}}\right) \\
& \cdot T\left[\left(-1+2 \gamma_{E}\right)+2 \log \left(\frac{2 m_{B}}{T}\right)\right] .
\end{aligned}
$$

The NLO contribution to the specific heat capacity is obtained as

$$
\begin{aligned}
& \left.C_{v}\right|_{\mathrm{NLO}} ^{B} \simeq\left(\frac{N_{C} N_{f}}{3}\right)\left(C_{f} \alpha_{s}\right)\left(m_{q}^{2} \frac{B}{B_{\mathrm{cr}}^{q}}\right) T\left[c_{1}\left(\frac{T}{m_{B}}\right)^{2 / 3}\right. \\
& \left.+c_{2}\left(\frac{T}{m_{B}}\right)^{4 / 3}+c_{3}\left(\frac{T}{m_{B}}\right)^{2}\left(c_{4}-\log \left(\frac{T}{m_{B}}\right)\right)\right] .
\end{aligned}
$$




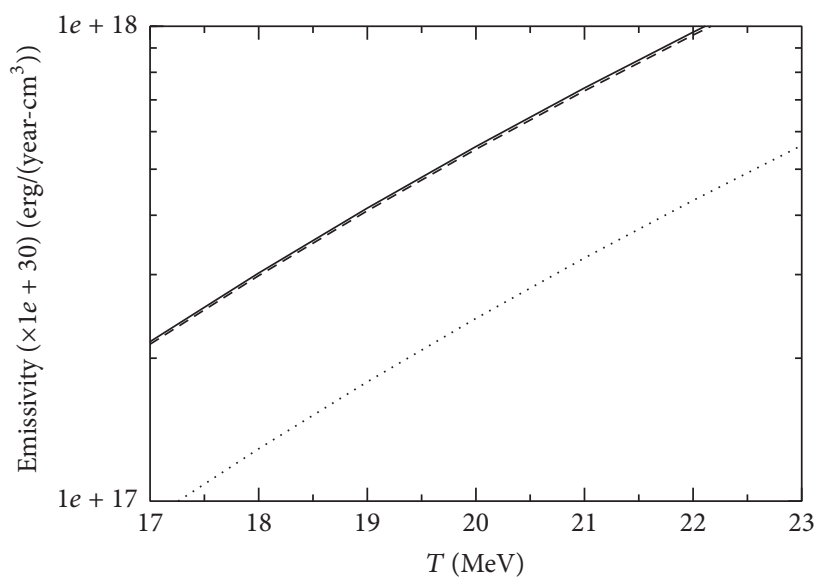

..... Without any correction (Fermi liquid result)

- - - With non-Fermi liquid correction up to LO

_ With non-Fermi liquid correction up to NLO

(a)

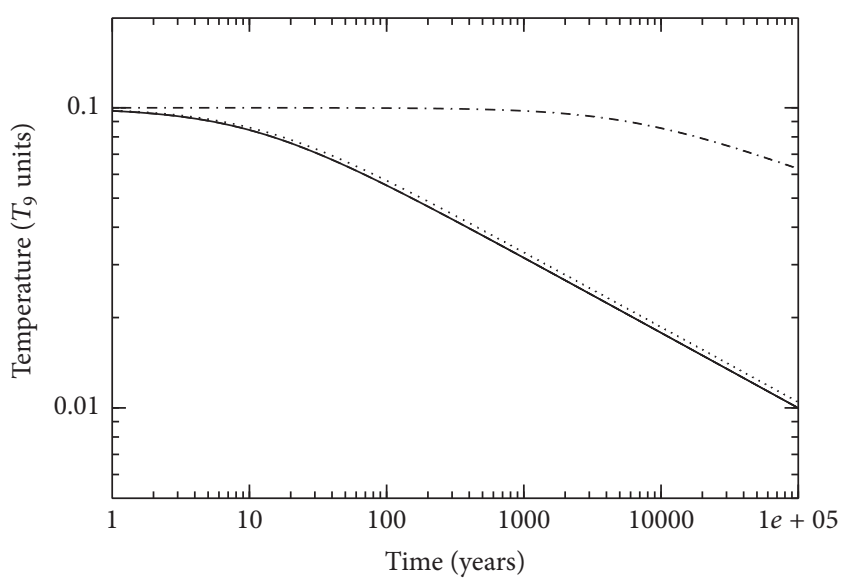

(b)

FIgURE 3: The emissivity of the neutrinos with temperature in degenerate quark matter $\left(T_{9}\right.$ in units of $\left.10^{9} \mathrm{~K}\right)$ has been shown in (a). The cooling behavior of neutron star with core as neutron matter and degenerate quark matter has been presented in (b). The dotted line represents the FL result, and the solid line depicts the NFL NLO correction. The dash-dotted line gives the cooling behavior of the neutron star core made up of purely neutron matter.

The in-medium propagators contain the dressed mass $m_{B}$ (Debye mass in the QCD case) in the presence of magnetic field:

$$
m_{B}^{2}=\frac{N_{f} g^{2} m_{q}^{2}}{4 \pi^{2}}\left(\frac{B}{B_{\mathrm{cr}}^{q}}\right) .
$$

The kick velocity taking into account the magnetic field effect on the specific heat capacity of the quarks reads as follows [34]:

$$
\begin{aligned}
\left.v\right|_{\mathrm{FL}} ^{B} \simeq & \frac{4.15 N_{C} N_{f}}{3}\left(\frac{\sqrt{m_{q}^{2}\left(B / B_{\mathrm{cr}}^{q}\right)}}{400 \mathrm{MeV}} \frac{T}{1 \mathrm{MeV}}\right)^{2} \\
& \cdot\left(\frac{R}{10 \mathrm{~km}}\right)^{3} \frac{1.4 M_{\odot}}{M_{\mathrm{NS}}} \chi \frac{\mathrm{km}}{\mathrm{s}} .
\end{aligned}
$$

With the incorporation of the anomalous effect, the LO contribution to the kick velocity is $[34,35]$

$$
\begin{aligned}
\left.v\right|_{\mathrm{LO}} ^{B} \simeq & \frac{8.8 N_{C} N_{f}}{3}\left(C_{f} \alpha_{s}\right)\left(\frac{\sqrt{m_{q}^{2}\left(B / B_{\mathrm{cr}}^{q}\right)}}{400 \mathrm{MeV}} \frac{T}{1 \mathrm{MeV}}\right)^{2} \\
& \cdot\left(\frac{R}{10 \mathrm{~km}}\right)^{3} \\
& \times \frac{1.4 M_{\odot}}{M_{\mathrm{NS}}}\left[0.0635+0.05 \log \left(\frac{m_{B}}{T}\right)\right] \chi \frac{\mathrm{km}}{\mathrm{s}}
\end{aligned}
$$

The calculation has been extended beyond the LO in NFL correction. The NLO correction is $[34,35]$

$$
\begin{aligned}
& \left.v\right|_{\mathrm{NLO}} ^{B} \simeq \frac{8.3 N_{C} N_{f}}{3}\left(\frac{B}{B_{\mathrm{cr}}^{q}}\right)\left(\frac{m_{q}}{400 \mathrm{MeV}} \frac{T}{1 \mathrm{MeV}}\right)^{2} \\
& \cdot\left(\frac{R}{10 \mathrm{~km}}\right)^{3} \frac{1.4 M_{\odot}}{M_{\mathrm{NS}}} \times \chi\left(C_{F} \alpha_{s}\right)\left[a_{1}\left(\frac{T}{m_{B}}\right)^{2 / 3}\right. \\
& +a_{2}\left(\frac{T}{m_{B}}\right)^{4 / 3} \\
& \left.+\left[a_{3}+a_{4} \ln \left(\frac{m_{B}}{T}\right)\right]\left(\frac{T}{m_{B}}\right)^{2}\right] \frac{\mathrm{km}}{\mathrm{s}} .
\end{aligned}
$$

The long range magnetic interactions are responsible for the anomalous $T^{2} \ln T^{-1}$ term in the expressions of the pulsar kick velocity.

For the case of cold neutron stars, the spin polarization of electrons is given by $[28,29]$

$$
\chi \simeq \frac{3}{2} \frac{m_{e}^{2}}{\mu_{e}^{2}-m_{e}^{2}}\left(\frac{B}{B_{\mathrm{cr}}^{e}}\right)
$$

where the critical value of the magnetic field is taken to be $B_{\mathrm{cr}}^{e} \simeq 4.4 \times 10^{13} \mathrm{G}$ to Landau-quantize the electrons. The weak magnetic field refers to $\left(\mu_{e}^{2}-m_{e}^{2}\right)>(2 e B)$ for partially occupied Landau levels which makes the spin polarization fraction $\chi \ll 1$. The second case arises when the strength of the magnetic field is chosen to be much larger than the temperature, the chemical potential, and the electron mass 

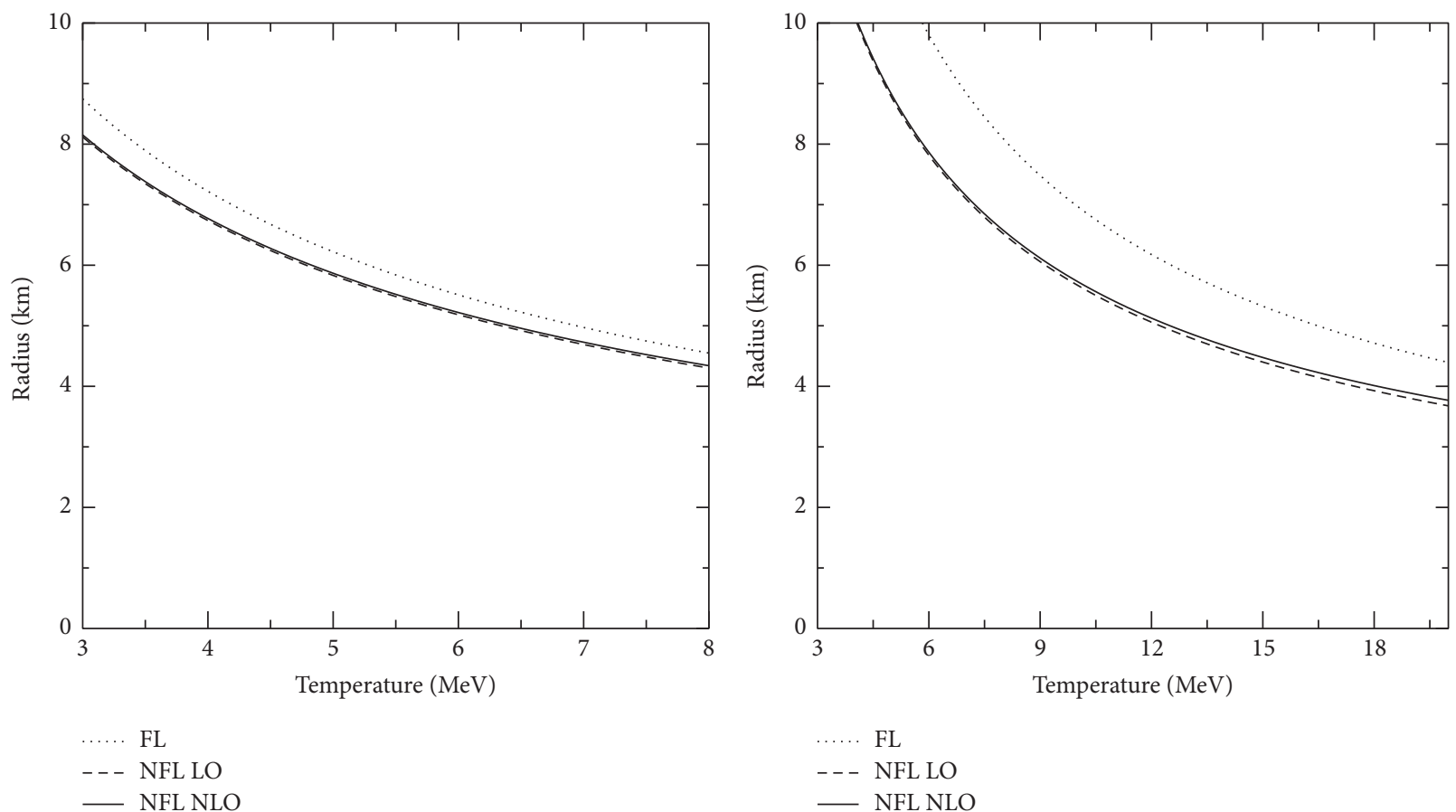

(a)

(b)

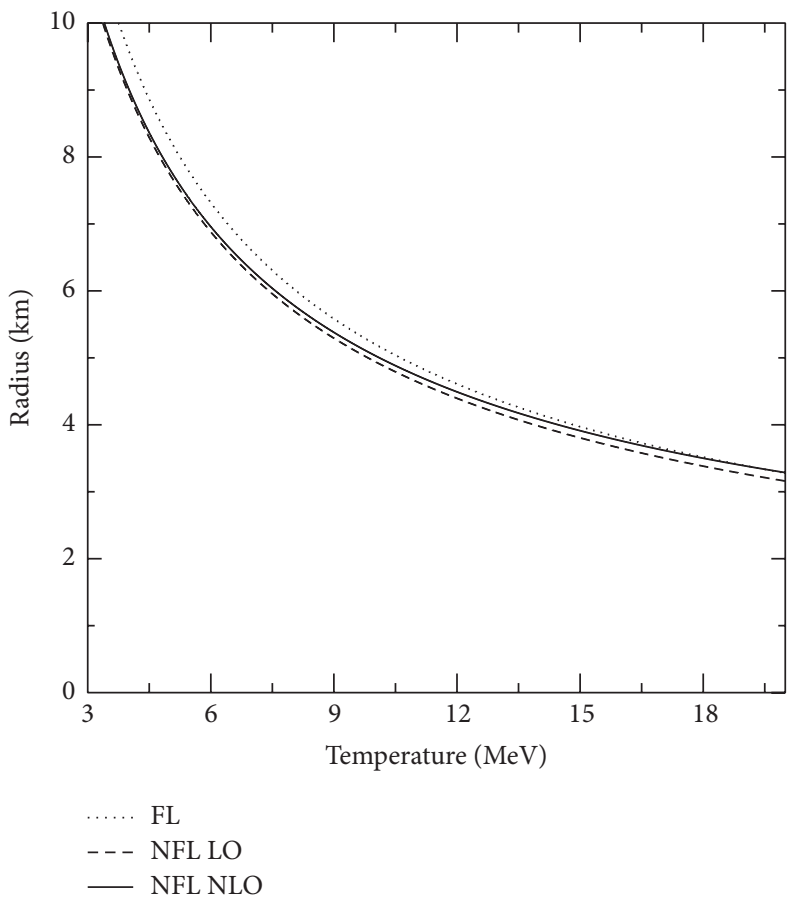

(c)

FIGURE 4: The comparison of the relationship (FL, LO, and NLO, resp.) where high magnetic field $\left(B=10^{19} \mathrm{G}\right)$ has been taken into account along with vanishing temperature for kick velocity of $100 \mathrm{~km} / \mathrm{s}$ has been shown in (a). (b) shows the corresponding case when high magnetic field effect in specific heat is included. (c) shows the comparison between the FL, NFL LO, and NFL NLO result for the radius and temperature dependence for partially polarized electrons in the presence of weak magnetic field $\left(B=5 \times 10^{15} \mathrm{G}\right)$ for kick velocity of $100 \mathrm{~km} / \mathrm{s}$. 
$\left(\mu_{e}, m_{e}, T \ll \sqrt{2 e B}\right)$ for $\chi$ close to unity. The electron polarization is given as [28]

$$
\chi \sim 1-\frac{4}{\ln (2)} \sqrt{\frac{\pi T}{2 \sqrt{2 e B}}} e^{-\sqrt{2 e B} / T} .
$$

For the case of large magnetic field, the kick velocity cannot be solved analytically. The net contribution to the pulsar kick velocity up to NLO is obtained by the sum of the Fermi liquid result and the non-Fermi liquid correction up to NLO:

$$
\left.v\right|_{\text {total }} ^{B}=\left.v\right|_{\mathrm{FL}} ^{B}+\left.v\right|_{\mathrm{LO}} ^{B}+\left.v\right|_{\mathrm{NLO}} ^{B} .
$$

With these results, an estimation of the quark phase radius of the NS with the temperature of the quark matter in the core is presented. In Figure 4, for the case of highly polarized electrons, a comparison between FL, LO, and NLO corrections to the kick velocity is shown in Figure 4(a). Figure 4(b) shows an exactly similar behavior with inclusion of the magnetic field on the specific heat capacity of quarks. In Figure 4(c), similar behavior for NFL cases is observed when the electrons are partially polarized for low magnetic fields.

It is observed that the pulsar kick velocity receives significant contribution from the logarithmic corrections at the LO in comparison to the FL result. The results are computed with LO and NLO corrections to include plasma or quasiparticle effects which are anomalous (NFL) effects. In addition, comparison has been made between the NFL LO and NLO contributions to the kick velocity with the FL case. It is seen that the NFL LO corrections are significant while calculating the radius-temperature relationship as seen in Figure 4. The anomalous corrections introduced to the pulsar kick velocity due to the NFL (LO) behavior are responsible for the appreciable increment of the kick velocity for a particular value of temperature found in the NS core. However, for all the cases, moderate change in the $R-T$ relationship has been observed for the NLO correction with respect to the LO case.

\section{Conclusions}

The prime objective of this present review is to present a comprehensive description of the collective excitations involving quasi-particles in the domain of relativistic dense plasma. It will not be out of context to recall that such ultradegenerate system is relevant for the astrophysical scenario of the QCD phase diagram (i.e., neutron stars). Thus, different physical quantities of interest have been studied for such system involving the quark direct URCA and quark-neutrino scattering reactions. We have found the MFP for both the degenerate and the nondegenerate neutrinos involving terms which have fractional powers in $(T / \mu)$ at higher orders using the perturbation theory and the NFL phenomenon as discussed above. In addition, we have also calculated the emissivity of neutrinos and examined NLO corrections over NFL LO and the simple FL case. Finally, we have examined the cooling behavior of the neutron star involving NLO correction to the emissivity of neutrinos as well as specific heat capacity of quark core. It might be noted that even though there is a modest correction to the quantities like MFP and emissivity of neutrinos over LO and FL case, there is a marginal alteration in the cooling behavior due to such NFL corrections. Although the NLO corrections are not quantitatively significant, these corrections at the higher order terms of the low temperature expansion involve fractional powers of $T$ as well as subsequent logarithmically enhanced term $T^{3} \ln (1 / T)$ which are important from the theoretical aspect of NFL formalism for temperature tending to zero. The dynamical screening along with Pauli blocking is a distinctive feature of cold and dense plasma unlike the case of high temperature.

The extension of the NFL phenomena to the study of pulsar kick velocities shows that the velocity receives significant contribution from the anomalous leading logarithmic correction. Further, incorporation of the modified quark dispersion relations up to the next-to-leading logarithmic order to the kick velocity has been studied. In addition, we have analyzed the effect of external magnetic field on the specific heat capacity of quarks which modifies the pulsar kick velocity. The primary mechanism for the generation of kick velocity is asymmetric neutrino emission. This asymmetry can be attributed to the strong magnetic field which can align the electron spin opposite to the magnetic field direction. Thus, we have taken into account the contribution from electron polarization fractions for different field strengths. We note that the presence of the logarithmic term and the fractional powers carrying signature of the weaker dynamical screening along with external magnetic field considerably enhances the kick velocity of the neutron star.

These results provide novel initiation for the study of the interplay of strong magnetic fields [66] with dense QCD matter which can have significant impact in the context of interesting astrophysical entities such as neutron star.

\section{Conflicts of Interest}

The author declares that there are no conflicts of interest regarding the publication of this paper.

\section{Acknowledgments}

The author would like to thank Professor T. K. Nayak for the continuous academic support during this work. The author would also like to thank Professor (Late) Abhee K. DuttMazumder and Professor P. K. Roy; without their supervision, this work would not have been possible.

\section{References}

[1] S. L. Shapiro and S. A. Teukolsky, Black Holes, White Dwarfs, and Neutron Stars, Wiley-VCH Verlag GmbH, Weinheim, Germany, 1983.

[2] A. B. Migdal, E. E. Saperstein, M. A. Troitsky, and D. N. Voskresensky, "Pion degrees of freedom in nuclear matter," Physics Reports, vol. 192, pp. 179-437, 1990.

[3] D. G. Yakovlev, A. D. Kaminker, O. Y. Gnedin, and P. Haensel, "Neutrino emission from neutron stars," Physics Reports, vol. 354, no. 1-2, pp. 1-155, 2001.

[4] D. N. Voskresensky, Neutrino Cooling of Neutron Stars: Medium Effects, vol. 578 of Lecture Notes in Physics, 2001. 
[5] D. Page, U. Geppert, and F. Weber, "The cooling of compact stars," Nuclear Physics A, vol. 777, pp. 497-530, 2006.

[6] A. Sedrakian, "The physics of dense hadronic matter and compact stars," Progress in Particle and Nuclear Physics, vol. 58, no. 1, pp. 168-246, 2007.

[7] X. G. Huang, Q. Wang, and P. F. Zhuang, "Neutrino emission from direct Urca processes in pion condensed quark matter," Physical Review D, vol. 76, no. 9, Article ID 094008, 2007.

[8] E. Witten, "Cosmic separation of phases," Physical Review D, vol. 30, no. 2, pp. 272-285, 1984.

[9] E. Farhi and R. L. Jaffe, "Strange matter," Physical Review D, vol. 30, no. 11, pp. 2379-2390, 1984.

[10] J. Gan and E. Wong, "Non-Fermi-liquid behavior in quantum critical systems," Physical Review Letters, vol. 71, no. 25, article 4226, 1993.

[11] S. Chakravarty, R. E. Norton, and O. F. Syljuåsen, "Transverse gauge interactions and the vanquished fermi liquid," Physical Review Letters, vol. 74, no. 8, pp. 1423-1426, 1995.

[12] M. Y. Reizer, "Relativistic effects in the electron density of states, specific heat, and the electron spectrum of normal metals," Physical Review B, vol. 40, no. 17, pp. 11571-11575, 1989.

[13] C. M. Varma, P. B. Littlewood, S. Schmitt-Rink, E. Abrahams, and A. E. Ruckenstein, "Phenomenology of the normal state of Cu-O high-temperature superconductors," Physical Review Letters, vol. 63, no. 18, pp. 1996-1999, 1989.

[14] J. Polchinski, String Theory, Cambridge University Press, Cambridge, UK, 1998.

[15] J. Polchinski, "Low-energy dynamics of the spinon-gauge system," Nuclear Physics B, vol. 422, no. 3, pp. 617-633, 1994.

[16] C. Nayak and F. Wilczek, "Renormalization group approach to low temperature properties of a non-Fermi liquid metal," Nuclear Physics B, vol. 430, no. 3, pp. 534-562, 1994.

[17] C. Manuel, "Dispersion relations in ultradegenerate relativistic plasmas," Physical Review D, vol. 62, no. 7, Article ID 076009, 2000.

[18] M. Le Bellac and C. Manuel, "Damping rate of quasiparticles in degenerate ultrarelativistic plasmas," Physical Review D, vol. 55, no. 5, pp. 3215-3218, 1997.

[19] T. Schäfer and K. Schwenzer, "Neutrino emission from ungapped quark matter," Physical Review D, vol. 70, no. 11, Article ID 114037, 2004.

[20] K. Pal and A. K. Dutt-Mazumder, "Non-Fermi liquid corrections to the neutrino mean free path in dense quark matter," Physical Review D, vol. 84, no. 3, Article ID 034004, 2011.

[21] A. Gerhold and A. Rebhan, "Fermionic dispersion relations in ultradegenerate relativistic plasmas beyond leading logarithmic order," Physical Review D, vol. 71, no. 8, Article ID 085010, 2005.

[22] T. Holstein, R. E. Norton, and P. Pincus, "De Haas-van Alphen effect and the specific heat of an electron gas," Physical Review $B$, vol. 8, no. 6, pp. 2649-2656, 1973.

[23] H. Heiselberg and C. J. Pethick, "Transport and relaxation in degenerate quark plasmas," Physical Review D, vol. 48, no. 6, pp. 2916-2928, 1993.

[24] S. Sarkar and A. K. Dutt-Mazumder, "Energy and momentum relaxation of heavy fermion in dense and warm plasma," Physical Review D, vol. 82, no. 5, Article ID 056003, 2010.

[25] S. Sarkar and A. K. Dutt-Mazumder, "Non-Fermi liquid behavior of the drag and diffusion coefficients in QED plasma," Physical Review D, vol. 84, no. 9, Article ID 096009, 2011.
[26] S. Sarkar and A. K. Dutt-Mazumder, "Non-Fermi liquid behavior of thermal relaxation time in degenerate electron plasma," Physical Review D, vol. 87, no. 7, Article ID 076003, 8 pages, 2013.

[27] A. Gerhold, A. Ipp, and A. Rebhan, "Non-Fermi-liquid specific heat of normal degenerate quark matter," Physical Review D-Particles, Fields, Gravitation and Cosmology, vol. 70, no. 10, Article ID 105015, 2004.

[28] I. Sagert, G. Pagliara, M. Hempel, and J. Schaffner-Bielich, "Is there quark matter in (low-mass) pulsars?” Journal of Physics G: Nuclear and Particle Physics, vol. 35, no. 10, Article ID 104079, 2008.

[29] I. Sagert and J. Schaffner-Bielich, "Pulsar kicks by anisotropic neutrino emission from quark matter in strong magnetic fields," A\&A, vol. 489, no. 1, pp. 281-289, 2008.

[30] D. Yakovlev and C. Pethick, "Neutron star cooling," Annual Review of Astronomy and Astrophysics, vol. 42, no. 1, pp. 169210, 2004.

[31] J. P. Finley, H. Ogelman, and U. Kiziloglu, "ROSAT observations of PSR $0656+14-$ a pulsating and cooling neutron star," The Astrophysical Journal, vol. 394, no. 1, pp. L21-L24, 1992.

[32] R. Anglani, G. Nardulli, M. Ruggieri, and M. Mannarelli, "Neutrino emission from compact stars and inhomogeneous color superconductivity," Physical Review D-Particles, Fields, Gravitation and Cosmology, vol. 74, no. 7, Article ID 074005, 2006.

[33] S. P. Adhya, P. K. Roy, and A. K. Dutt-Mazumder, "Next-to-leading order non-Fermi-liquid corrections to the neutrino emissivity and cooling of the neutron star," Physical Review D, vol. 86, no. 3, Article ID 034012, 2012.

[34] S. P. Adhya, P. K. Roy, and A. K. Dutt-Mazumder, "Modifications to the pulsar kick velocity due to magnetic interactions in dense plasma," Journal of Physics G: Nuclear and Particle Physics, vol. 41, no. 2, Article ID 025201, 2014.

[35] S. P. Adhya and P. K. Roy, "Role of magnetic interactions in neutron stars," EPJ Web of Conferences, vol. 95, Article ID 04001, 8 pages, 2015.

[36] N. Iwamoto, "Neutrino emissivities and mean free paths of degenerate quark matter," Annals of Physics, vol. 141, no. 1, pp. $1-49,1982$.

[37] J. M. Lattimer, C. J. Pethick, M. Prakash, and P. Haensel, "Direct URCA process in neutron stars," Physical Review Letters, vol. 66, no. 21, pp. 2701-2704, 1991.

[38] D. L. Tubbs and D. N. Schramm, "Neutrino opacities at high temperatures and densities," The Astrophysical Journal, vol. 201, pp. 467-488, 1975.

[39] D. Q. Lamb and C. J. Pethick, "Effects of neutrino degeneracy in supernova models," The Astrophysical Journal, vol. 209, pp. L77-L81, 1976.

[40] K. Sato and T. Tatsumi, "Spontaneous magnetization in QCD and non-Fermi-liquid effects," Nuclear Physics A, vol. 826, no. 1-2, pp. 74-100, 2009.

[41] S. P. Adhya, P. K. Roy, and A. Dutt-Mazumder K, "Non-Fermi liquid correction to the neutrino mean free path and emissivity in neutron star beyond the leading order," AIP Conference Proceedings, vol. 1524, pp. 263-266, 2013.

[42] M. Alford, K. Rajagopal, and F. Wilczek, "Color-flavor locking and chiral symmetry breaking in high density QCD," Nuclear Physics B, vol. 537, no. 1-3, pp. 443-458, 1999.

[43] M. Huang, "Color superconductivity at moderate baryon density," International Journal of Modern Physics E, vol. 14, p. 675, 2005. 
[44] I. Shovkovy and M. Huang, "Gapless two-flavor color superconductor," Physics Letters B, vol. 564, no. 3-4, pp. 205-211, 2003.

[45] M. Huang and I. Shovkovy, "Gapless color superconductivity at zero and at finite temperature," Nuclear Physics A, vol. 729, no. 2-4, pp. 835-863, 2003.

[46] M. Alford, C. Kouvaris, and K. Rajagopal, "Gapless color-flavorlocked quark matter," Physical Review Letters, vol. 92, no. 22, Article ID 222001, 2004.

[47] M. Alford, J. A. Bowers, and K. Rajagopal, "Crystalline color superconductivity," Physical Review D, vol. 63, no. 7, Article ID 074016, 2001.

[48] M. Iwasaki and T. Iwado, "Superconductivity in quark matter," Physics Letters B, vol. 350, no. 2, pp. 163-168, 1995.

[49] R. D. Pisarski and D. H. Rischke, "Color superconductivity in weak coupling," Physical Review D, vol. 61, no. 7, Article ID 074017, 2000.

[50] M. Schäfer, "Ergebnisse der Kariesrisikobetreuung unter soziodemographischen Aspekten," Das Gesundheitswesen, vol. 62, no. 11, pp. 589-592, 2000.

[51] A. Schmitt, Q. Wang, and D. H. Rischke, "When the transition temperature in color superconductors is not like in BCS theory," PRD, vol. 66, Article ID 114010, 2002.

[52] A. Schmitt, Q. Wang, and D. H. Rischke, "Electromagnetic Meissner effect in spin-one color superconductors," Physical Review Letters, vol. 91, no. 24, Article ID 242301, 4 pages, 2003.

[53] M. G. Alford, J. A. Bowers, J. M. Cheyne, and G. A. Cowan, "Single color and single flavor color superconductivity," Physical Review D, vol. 67, no. 5, Article ID 054018, 2003.

[54] A. Schmitt, "Ground state in a spin-one color superconductor," Physical Review D, vol. 71, no. 5, Article ID 054016, 2005.

[55] M. Alford, P. Jotwani, C. Kouvaris, J. Kundu, and K. Rajagopal, "Astrophysical implications of gapless color-flavor locked quark matter: a hot water bottle for aging neutron stars," Physical Review D, vol. 71, no. 11, Article ID 114011, 2005.

[56] A. Schmitt, I. A. Shovkovy, and Q. Wang, "Neutrino emission and cooling rates of spin-one color superconductors," Physical Review D, vol. 73, no. 3, Article ID 034012, 2006.

[57] P. Jaikumar, C. D. Roberts, and A. Sedrakian, "Direct Urca neutrino rate in color superconducting quark matter," Physical Review C, vol. 73, no. 4, Article ID 042801, 2006.

[58] R. Anglani, G. Nardulli, M. Ruggieri, and M. Mannarelli, "Neutrino emission from compact stars and inhomogeneous color superconductivity," Physical Review D, vol. 74, no. 7, Article ID 074005, 13 pages, 2006.

[59] Q. Wang, Z. G. Wang, and J. Wu, "Phase space and quark mass effects in neutrino emissions in a color superconductor," Physical Review D, vol. 74, no. 1, Article ID 014021, 2006.

[60] G. W. Carter and S. Reddy, "Neutrino propagation in color superconducting quark matter," Physical Review D, vol. 62, no. 10, Article ID 103002, 2000.

[61] P. Jaikumar and M. Prakash, "Neutrino pair emission from Cooper pair breaking and recombination in superfluid quark matter," Physics Letters B, vol. 516, no. 3-4, pp. 345-352, 2001.

[62] D. Yakovlev and C. Pethick, "Neutron Star Cooling," Annual Review of Astronomy and Astrophysics, vol. 42, no. 1, pp. 169210, 2004.

[63] R. E. Rutledge, L. Bildsten, E. F. Brown, G. G. Pavlov, and V. E. Zavlin, "The quiescent $\mathrm{x}$-ray spectrum of the neutron star in centaurus x-4 observed with Chandra/ACIS-S," The Astrophysical Journal, vol. 551, no. 2, pp. 921-928, 2001.
[64] S. Chakrabarty, "Quark matter in a strong magnetic field," Physical Review D, vol. 54, no. 2, pp. 1306-1316, 1996.

[65] D. Bandopadhyay, S. Chakraborty, and S. Pal, "The Quantizing magnetic field and quark-hadron phase transition in a neutron star," Physical Review Letters, vol. 79, p. 12, 1997.

[66] S. P. Adhya, M. Mandal, S. Biswas, and P. K. Roy, "Pionic dispersion relations in the presence of a weak magnetic field," Physical Review D, vol. 93, no. 7, Article ID 074033, 2016. 

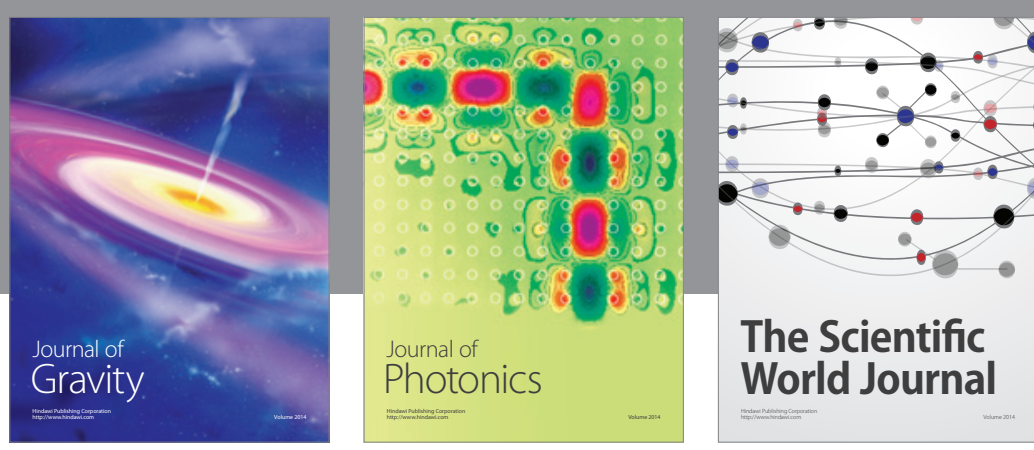

The Scientific World Journal
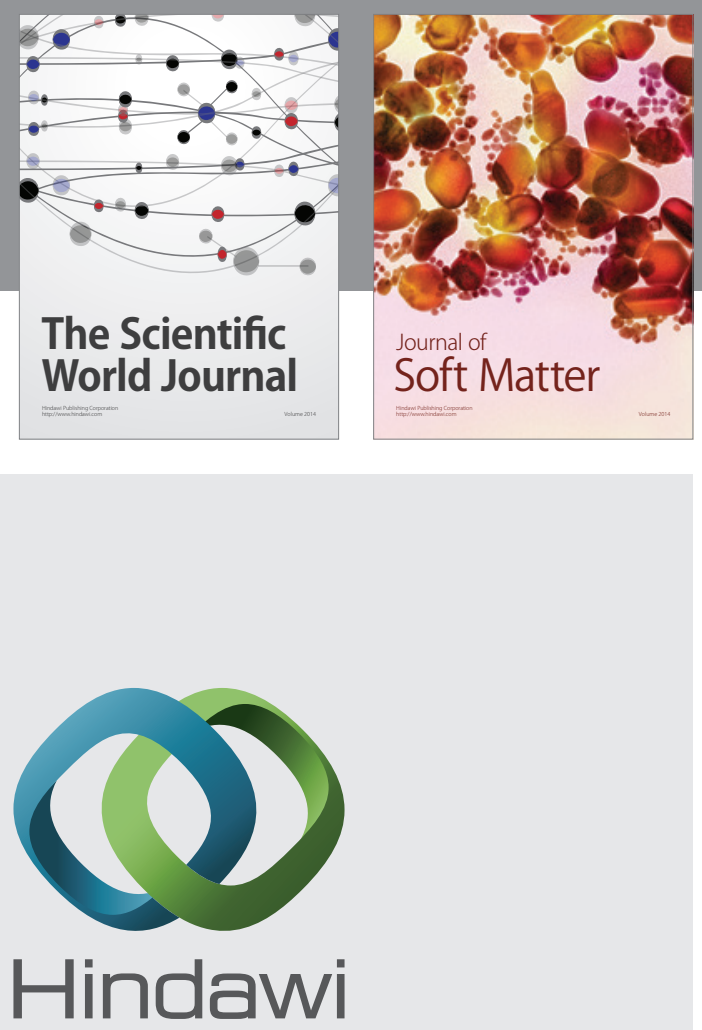

Submit your manuscripts at

https://www.hindawi.com
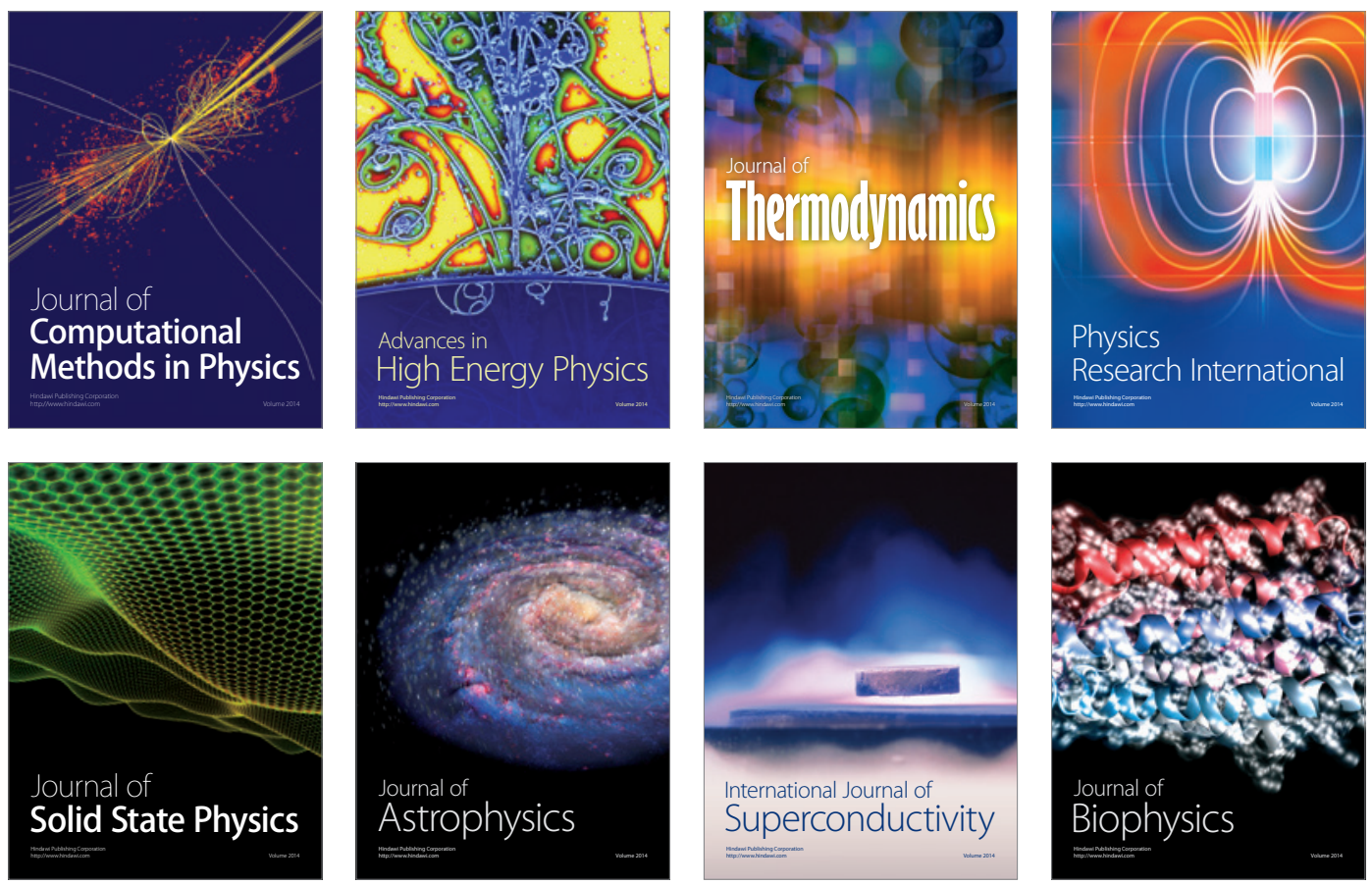
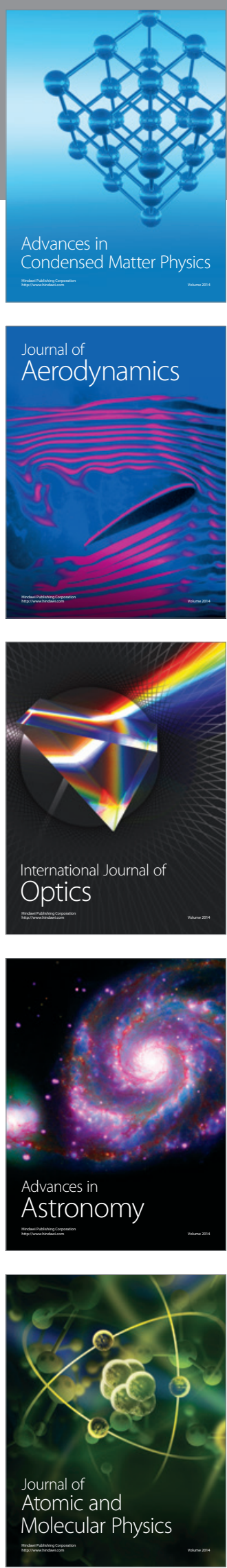